\title{
Gerencia, cultura organizacional y gestión del conocimiento en la administración financiera de las pequeñas y medianas empresas (PYMES) en Venezuela
}

\author{
Orlando Canelones', Williams Aranguren², Paola Lamenta ${ }^{3}$ \\ Fecha de recepción: 25 de Febrero 2018 \\ Fecha de aceptación: 28 de Marzo 2018
}

\section{Resumen}

El objetivo de esta investigación es establecer una visión general de un aspecto fundamental de la gerencia, la cultura organizacional y la gestión del conocimiento dentro de la administración financiera, como lo es el acceso al financiamiento de las pequeñas y medianas empresas (PYMES) en Venezuela, siendo este último un factor primordial para la gerencia. En este sentido, se realiza un abordaje documental desde diversas ópticas, con el propósito de visualizar un alto grado de cultura organizacional que se manifiesta en la gestión del conocimiento; ya que estas herramientas gerenciales pueden coadyuvar en cualquier momento que sea requerido por la gerencia en el acceso a los recursos financieros que puedan requerir las PYMES. Se puede concluir que las PYMES en Venezuela, al igual que en muchos países latinoamericanos, presentan dificultades para su supervivencia, por lo que requieren ser fortalecidas en el tiempo con técnicas y planes de financiamiento, partiendo de potenciar sus capacidades gerenciales y fortificando su cultura organizacional.

Palabras clave: Cultura Organizacional, Gestión del conocimiento, Gerencia, Financiamiento, PYMES.

\section{Abstract}

The objective of this research is to establish a general vision of a fundamental aspect of management, organizational culture and knowledge management within financial administration, such as access to financing for small and medium enterprises (SMEs) in Venezuela. , the latter being a key factor for management. In this sense, a documentary approach is made from different perspectives, with the purpose of visualizing a high degree of organizational culture that is manifested in knowledge management; since these management tools can contribute at any time that is required by management in accessing the financial resources that may be required by SMEs. It can be concluded that SMEs in Venezuela, as in many Latin American countries, present difficulties for their survival, so they need to be strengthened over time with financing techniques and plans, based on strengthening their managerial capacities and strengthening their organizational culture.

Keywords: Organizational Culture, Knowledge Management, Management, Financing, SMEs.

\section{Introducción}

La economía venezolana actualmente se caracteriza por una transformación de los hechos y fenómenos económicos que afectan significativamente a las organizaciones, en todos sus niveles, tales como inflación elevada, contracción económica, control de cambio, entre otras, lo cual genera inestabilidad y a lo que las pequeñas y medianas empresas (PYMES) son susceptibles.

La mayoría de las PYMES en Venezuela son unidades de producción de tipo familiar, que centran sus intereses principalmente en la gestión del negocio y en los sistemas financieros-contables. Se basan en valores éticos compartidos más que en la competencia con otras empresas, caracterizándose por poseer una estructura rígida y muy tradicional. Mercado y Palmerín (2007) destacan algunas de las principales características de las PYMES, entre las cuales se mencionan: la producción es el centro de la vida empresarial, liderazgo autocrático, limitaciones en el acceso a indicadores de gestión y de control administrativo, carácter rentista (dependiente de políticas gubernamentales), ausencia de gestión del conocimiento como estrategia gerencial y comportamiento gerencial mecanicista.

Complementando lo anterior, en el contexto Venezolano, las PYMES representan en promedio más del $90 \%$ de los establecimientos del país y han venido reduciendo su número progresivamente, empleando al $36 \%$ de la mano de obra ocupada según datos de la Encuesta Industrial (INE, 2018). Con relación a la productividad, las PYMES igualmente evidencian un decrecimiento del valor bruto de producción (VBP), con un aporte del 14\% del total en promedio en los años 2000-2007 y para el año 2008 no representa más del 12\%. Estas empresas tienen poca participación en los ingresos de la nación, representando en promedio apenas el 13,52\% del valor total de las ventas y el 3\% de las exportaciones, dedicándose casi exclusivamente a la producción local y regional. En resumen, las PYMES en Venezuela es el segmento de mayor representación del país, aunque en cifras no lo reflejan en cuanto a inversión, exportación, ocupación, entre otras.

Conforme a lo anterior, las instituciones financieras privadas y públicas tratan en lo posible de contribuir en la disminución del problema al acceso de financiamiento de las pequeñas y medianas empresa (PYMES). Al respecto Canelones (2014) destaca que las PYMES encuentran limitaciones en el acceso al crédito, representando uno de los principales obstáculos para su consolidación y desarrollo, siendo que a pesar de estas limitaciones, los gerentes deben poseer la tenacidad y formación para buscar mejores garantías, en la cual la iniciativa empresarial salga fortalecida en la prosecución de los fondos necesarios para cimentar y desarrollar las PYMES.

Por su parte Galindo (1999) plantea que las PYMES presentan determinados impedimentos cuyas consecuencias no han sido consideradas en los distintos productos y operaciones financieras, y que también se han dedicado esfuerzos a elogiar las cualidades de las tradicionales fórmulas de financiación, sin atender a la efectividad de su aplicación potencial. Esta realidad hace necesario e imprescindible la generación de ideas o soluciones destinadas a implementar esfuerzos para que los gerentes de las empresas establezcan la adaptación a nuevas situaciones, dándole la importancia requerida, siendo indispensable para las PYMES, si se desea conseguir la supervivencia en esta época tan vulnerable al cambio en la administración financiera.

Bajo este planteamiento Cárdenas, González, González y Marín (2008:575) indican que:

A medida que los sistemas financieros se van desarrollando y tiene lugar el proceso de intervención de las economías de los distintos países, formando un mercado financiero internacional, surge la necesidad de estudiar con rigor la intensidad de la incidencia de determinados elementos del entorno sobre las principales magnitudes o indicadores de la situación financiera de las empresas.

Con base en lo anterior, el trabajo de investigación representa una contribución al conjunto de conocimientos sobre el tema, ya que el mismo proporciona a las pequeñas y medianas empresas en Venezuela y Latinoamérica, herramientas que les permitan mantenerse a flote. También la investigación permite visualizar antagonismos de carácter sistemático

Economista. Magister en Administración de Empresas. Docente Agregado de la Facultad de Ciencias Económicas y Sociales de la Universidad de Carabobo. Doctorando en Ciencias Administrativas y Gerenciales de la Universidad de Carabobo. Email: ocanelones@gmail.com

2Sociólogo. Doctor en Ciencias Sociales Mención Estudios del Trabajo. Especialista en Seguridad Social Mención Gerencia Social. Docente Asociado de la Facultad de Ciencias Económicas y Sociales de la Universidad de Carabobo. Email: waranguren30@gmail.com

3 Licenciada en Contaduría Pública. Magíster en Administración de Empresas Mención Gerencia. Docente Asociado de la Facultad de Ciencias Económicas y Sociales de la Universidad de Carabobo. Doctorando en Gerencia Universidad de Yacambú. Email: paolalamenta@gmail.com 
y de naturaleza práctica para los gerentes de las pequeñas y medianas empresas, con la única finalidad de mejorar su gestión del conocimiento y la eficacia de sus firmas a la vez que busca determinar como la gerencia, a través de la gestión del conocimiento y la cultura organizacional dentro de la administración financiera, son herramientas imprescindibles en el acceso del financiamiento de la pequeña y mediana empresa.

\section{Metodología}

La orientación metodológica considerada fue la cualitativa, basada en un pluralismo metodológico integrado, que permitió la aplicación de técnicas y procedimientos operativos cualitativos para conocer las relaciones que se establecen, las contradicciones y conflictos que se producen así como los continuos cambios generados en los documentos objeto de estudio, vistos éstos como una totalidad. En consecuencia, la ruta metódica seguida es el método hermenéutico como estudio de teoría y práctica del entendimiento, en particular en la interpretación de significados. La técnica utilizada fue el análisis de contenido. En este sentido, se realizó la recolección de la información a través de la selección del instrumento como: libros, artículos, estadísticas oficiales, entre otros donde se encuentran las directrices de la temática abordada.

\section{Resultados}

\section{1.- La Gerencia}

La gerencia en las PYMES no difiere en gran medida de lo que ocurre en todas las organizaciones venezolanas, prevaleciendo elementos vinculados con la vertiente tradicional de la gerencia caracterizada por Aranguren (2009:7) de la siguiente manera:

...mantener la tradición en la gestión (administrativa, de recursos humanos, etc.), resistencia a los cambios dentro de la organización (trata de mantener lo aprendido con poca o ninguna innovación), énfasis en lo factible (no se arriesga en proyectos osados), poco flexible (se enmarca dentro de las normas y procedimientos establecidos no aceptando la disidencia), limitada planeación (muchas veces existe sólo a nivel formal), reacciona frente a los hechos ocurridos (no se adelanta a los acontecimientos) y el trabajo es directivo (prevalece la jerarquía de mando), entre muchos otros aspectos.

De lo anterior se desprende que el éxito o fracaso de una organización es responsabilidad de la gerencia, para coordinar los esfuerzos en la consecución de los objetivos institucionales, realizando las funciones de planificación, organización, dirección y control, como un sistema efectivo con interdependencia de estas.

De lo citado y referido de este autor, se desprende que la práctica gerencial requiere de la participación de un gerente, artífice de la gerencia en las organizaciones. No se puede negar las bondades que ha aportado la gerencia tradicional al proceso de organización y funcionamiento de las empresas, no obstante, hoy en día las empresas son cada vez menos estructuradas y éstas funciones que se emplean bien en ambientes normativos, van dejando déficits en la gerencia que deben ser tratados para mantenerse a flote en el mercado.

En este aspecto el gerente cumple con un papel fundamental, tal como lo establece Hernández (2005), pues es a quien corresponde dirigir las fases de mantenimiento de la institución, administrando el orden organizacional, la implantación de sistemas y procedimientos, la coordinación y el control. Lo anterior permite reflexionar acerca de la actitud profesional del gerente, el cual requiere adaptarse a los cambios que le permitan planificar los mismos y se presenten en el mundo de hoy con el único objetivo de obtener la máxima excelencia organizacional.

Por su parte Romero (2009:17) expone que: “...el gerente deberá contextualizar sus conocimientos sin desprenderse de la postura epistemológica que le es inmanente en el plano de la realidad de la que forma parte". El gerente, como pieza clave en una organización en constante cambio, va a necesitar de su pericia y tomar en cuenta todas las variables que conlleven a la mejor toma de decisiones. Es decir, cuando el gerente solo toma en cuenta los elementos de tipo económico y financiero cuantificables, muchas veces sus conclusiones son distorsionadas y se distancian de la realidad. En este caso, puede pensarse que la apreciación del gerente no fue la mejor, pero al final, sucede que la toma de decisión definitiva no involucra elementos de juicio que no se encuentran en el análisis económico de alternativas y por ende los resultados pueden no ser los esperados. De allí la imperiosa necesidad de visualizar el contexto en el cual se desarrollan las organizaciones, de una manera integral, global, desde diferentes miradas.

Ramos (2014) explica la importancia del desarrollo y fortalecimiento del conocimiento desde un enfoque sistémico en beneficio de la colectividad, para una mejor comprensión de lo social y las necesidades humanas.

Sobre este aspecto destaca la relevancia de la gerencia de recursos humanos que según Sotomayor (2004:.81) tiene por finalidad "lograr que los trabajadores contribuyan a alcanzar las metas de la organización, mientras directa o indirectamente van a satisfacer sus necesidades individuales o colectivas". Lo anterior implica contar con un personal involucrado con los objetivos de la empresa, que de acuerdo con Márquez (1998), debe ser capacitado y multifuncional, contribuyendo al éxito organizacional y a enfrentar los desafíos que hoy en día se perciben en la competencia mundial.

La capacidad de adaptación y la disposición al cambio en las personas debe ser un requisito indispensable, así como también disponer de personal que maneje la tecnología y los avances en forma expedita, siendo prioritario disponer de una gerencia con un alto estándar de conocimientos y cultura organizacional, que en el momento de necesidad financiera coadyuve a la consecución de los recursos. La gerencia deba ser dinámica, flexible, abierta a los posibles escenarios que plantean los retos de hoy, manejar la incertidumbre y lo que se ha dado en llamar la desorganización creativa, que no es más que la descomposición de las estructuras tradicionales de las empresas y sus formas de funcionamiento, para poder adaptarse y desenvolverse en un mundo que cada día envía señales de mayor complejidad y requerimientos de adaptabilidad al contingente.

\section{2.- Gerencia y cultura organizacional}

A través de los años se han realizado estudios acerca de la cultura organizacional, las relaciones humanas y su influencia en la gerencia, donde resalta la importancia que ésta representa para las organizaciones. Freitas (1991) explica el tema de cultura organizacional, sugiriendo que la investigación llevada a cabo en esta área representa en gran medida las respuestas a los problemas prácticos identificados por la gerencia, en el cual su estudio se ha traducido en un proceso que incluye una falta de uniformidad y cohesión de los patrones culturales. La cultura organizacional emerge en un contra-ataque a los problemas de la desintegración de la sociedad, como una solución atractiva, haciendo hincapié en las ideas comunes, formas de pensamiento, valores, normas y formas de trabajar.

Sobre este particular García (2006) afirma que la cultura se concibe como un elemento que influye invariablemente en las organizaciones, pues la misma es nutrida por las múltiples experiencias vividas por cada uno de sus integrantes. Por lo tanto, a los gerentes les corresponde buscar en el contexto de la organización, las metas y objetivos e internalizarlos de una forma muy amplia, con el objeto de que forme parte de su cultura organizacional y ponerlo en práctica haciendo hincapié en el éxito de la organización.

Todo lo anterior puede influir en el desarrollo adecuado de la gestión gerencial, tal como lo señalan Nonaka y Takeuchi (1999) cuando expresan que los estudios de la cultura organizacional han destacado la importancia de los valores, símbolos y creencias dentro de la organización, vista como un sistema de significados compartidos, que puede aprender, cambiarse a sí misma y evolucionar en el tiempo. Adicionalmente, estos autores distinguen dos dimensiones en el proceso de creación del conocimiento: la epistemológica representada por el 
conocimiento explícito y tácito, convirtiéndose en un espiral del conocimiento a través de la interacción dinámica entre las diferentes modalidades de conversión del conocimiento; y la ontológica que distingue cuatro niveles de agentes creadores del conocimiento: el individuo, el grupo, la organización y el nivel inter-organizativo, todo esto como parte de la cultura organizacional. Celis (2015) agrega que la cultura organizacional constituye un modelo complejo donde se involucran creencias, valores, actitudes y conductas que son compartidas por los integrantes de la organización que evolucionan con el paso del tiempo.

Por su parte, Freitas (1991) sostiene que la misma cultura organizacional proporciona un sin número de respuestas a las tendencias de la descomposición social, estableciéndose la ruptura de los patrones culturales, que reproduce un orden en particular, mediante el consenso y la solidaridad entre los miembros de la organización. Al tratar estos aspectos, se evidencia que existe una mezcla de factores sociales y culturales que pueden servir de apoyo a la organización que quizás pueda resultar difícil pero no imposible, ya que el mismo medio organizacional representado en la cultura de la organización, conlleva a la adaptación general de todos sus miembros y a su vez encaminarse a la búsqueda de objetivos comunes.

Lo expuesto anteriormente resalta el señalamiento de Briceño (2011), quien afirma que la cultura grupal es el resultado de procesos de interacción complejos (valores, creencias y actitudes), cuya existencia puede inferirse a partir de las conductas, lo cual representaría lo único susceptible de ser estudiado. Esta postura, proveniente del interaccionismo simbólico, permite inferir que al estudiar la conducta de la gente se concibe una exploración del comportamiento humano y sus condiciones.

De lo anteriormente expuesto, se percibe como eje trasversal la idea del cambio en las organizaciones, como una especie de imperativo que evoluciona independientemente de las voluntades humanas. En este orden Valera (2011) expresa la necesidad de transformar la mentalidad de las personas para generar el cambio, a través de la tecnología y la búsqueda de nuevos y mejores sistemas de vida.

De esto se desprenden las bases que asentarán al gerente de hoy, lo cual hace necesario expresar lo dicho por Drucker (2002: 89-90):

El cambio no se puede administrar. Solamente podemos mantenernos delante de él. Pero si una organización, sea un negocio, una universidad, un hospital, entre otras, no ve como su cometido liderar el cambio, esa organización no va a sobrevivir. Hay que tener políticas encaminadas a que el presente sea forjador del futuro.

Es por ello, que la cultura organizacional cobra importancia en los seres humanos, ya que la misma representa sus ideas, valores, actitudes y acciones que pueden ejercer de forma apropiada en el ser humano, conductas gerenciales que coadyuven a las organizaciones a lograr el éxito y metas planteadas. Todo esto, constituye y contribuye a generar, construir las bases necesarias de la gestión del conocimiento, el cual se construye a través de las prácticas cotidianas de vida que generan cultura, fundamental a la hora de conocer (León, 2014).

Gálvez y García (2011: 141) confirman que las investigaciones muestran que los valores y las prácticas organizacionales asociadas a la cultura de innovación, forjan mejoras en la eficacia de las personas, lo cual se manifiesta en la calidad de los productos y servicios y en la relación de la empresa con su entorno. Ello permite deducir que la cultura organizacional dentro de la administración financiera representa una variable o factor ineludible en el acceso del financiamiento de las PYMES.

Teniendo una cultura organizacional dentro de los parámetros de mejorar la capacidad de trabajar en equipo, la aceptación del riesgo y poder manejar la incertidumbre, implica de forma notoria la mejora del desempeño de la organización y se puede afrontar con éxito la falta de recursos en un momento dado por parte de las PYMES, para llevar a cabo los proyectos de expansión o inversión.

\section{3.- Gerencia y la gestión del conocimiento}

En la actualidad, el rol de la gestión del conocimiento en las organizaciones ha venido cambiando debido a la aparición de nuevos paradigmas asociados al mismo, es por ello por lo que las organizaciones buscan de cualquier forma estar a la par de las necesidades cambiantes del mercado y poder con ello lograr satisfacer dichas necesidades. Barrigaz y Bravo (2012) establecen que para una empresa, la gestión del conocimiento debe estar centrada en el hecho de que permite crear ventajas competitivas, y así poder enfrentar una serie de aspectos que existen en la actualidad en el contexto donde desarrolla sus actividades. Lo señalado por estos autores, resalta la importancia de la gestión del conocimiento en cualquier organización y cobra más jerarquía cuando el enfoque va dirigido a las PYMES.

Pedraja, Rodríguez y Rodríguez (2009: 497) afirman que:

Las pequeñas y medianas empresas son organizaciones que representan más del $80 \%$ del total de empresas que existen en Latinoamérica. Además, estas empresas son las que contribuyen en mayor medida a la generación de empleo y a la dinámica que se logra en las economías emergentes. Por lo tanto, descubrir si la gestión del conocimiento impacta sobre la eficacia de este tipo de empresas puede contribuir a su mejoramiento y, por ende, al crecimiento de un sector económico importante en los países en vías de desarrollo.

Esta visión de gestión del conocimiento en las PYMES, va a dar impulso a la generación, almacenaje y transmisión del conocimiento en las organizaciones, en un contexto emergente, sirviendo de filtro a los gerentes para la toma de decisiones cuando surge la necesidad del saber. Como lo expone Morín (2000), la educación debe despejar las dudas sobre la posibilidad de conocer, pues para las organizaciones, estas interrogantes constituyen el oxígeno para cualquier empresa de conocimiento.

Esta idea guarda relación con lo planteado por Buckingham y Coffman (1999), quienes expresan que el conocimiento es sencillamente aquello de lo cual la persona tiene conciencia y este conocimiento puede ser objetivo, que son las cosas que la persona sabe y vive; lo cual es algo diferente, ya que se aprende a lo largo del camino, es responsabilidad de cada uno. Es aquí, donde el conocimiento y el ser humano, adquieren forma y significado, dado la importancia del conocer, porque si se toman iniciativas o propuestas educativas del mundo de hoy como punto de partida, se pueden formular propuestas que den soluciones a las organizaciones.

Por otro lado, León (2014:20) explica que la teoría del conocimiento, como doctrina filosófica, distingue cuatro elementos: el sujeto que conoce, el objeto conocido, la operación misma de conocer y el resultado obtenido, que es la información que se recaba sobre el objeto. Dicho de otra manera: el sujeto se pone en contacto con el objeto y obtiene una información acerca del mismo. Cuando existe congruencia o adecuación entre el objeto y la representación interna correspondiente, se dice que posee la verdad. Al analizar lo planteado anteriormente, se puede decir que quien posee la verdad está en posesión de conocimiento y a su vez tiene la capacidad de poder gestionar conocimiento. Así lo plantean Pedraja, Rodríguez. y Rodríguez. (2009), quienes manifiestan que el conocimiento en cualquier campo, otorga poder, capacidad de acción y decisión a quienes lo poseen, de allí la importancia en una organización de gestionar el conocimiento basada en la eficiencia organizacional, lo cual impactará positivamente sobre la rentabilidad económica de la empresa.

Para León (2014), en el ámbito gerencial y dirección de empresas, cuando se hace referencia a la eficacia organizativa, ésta va a estar asociada tradicionalmente a medidas de orden económico y financiero. Es aquí donde la gestión del conocimiento puede convertirse en un principio esencial de ventaja competitiva dentro de la economía de la información, para lo cual se hace primordial que las PYMES establezcan sistemas eficientes de información, tanto internos como externos, y dicha información debe ser eficientemente procesada y puesta en práctica. Para Briceño (2011), el capital humano y su desarrollo, conforman elementos fundamentales en las sociedades basadas en el conocimiento, necesario 
para el desarrollo humano. Ante este planteamiento, el autor refleja la necesidad de realizar un manejo eficaz de la información, la cual va a ser precisa para la excelencia, la competitividad y la toma de decisiones financieras de las PYMES, que influirán en el desarrollo social y cultural de las personas.

Cabe destacar, que el manejo de la gestión del conocimiento se vuelve cada vez más complejo, debido a la explosión de la oferta y la demanda de información y al desarrollo de tecnología de información y comunicación en el campo de la telemática durante los últimos años. Al respecto se destaca a Mujica (2010), indicando que los grandes cambios de paradigmas gerenciales van a la par de la tecnología y que cada día la administración y lo tecnológico se hacen mutuamente incluyentes y complementarios. Esto concuerda con lo expresado por Villagrasa (2011), quien propone un cambio organizacional orientado hacia la infomacionalización, a través del desarrollo de las tecnologías de la información y comunicación, abriendo paso a un modelo económico y social emergente.

En el Cuadro 1, se refleja el conjunto de tecnologías que habilitan la gestión del conocimiento realizado por Villagrasa (2011) a partir de la información de Bulchand (2002), quien describe de manera precisa algunas concepciones al respecto, con las respectivas consideraciones y propósito o utilidad de cada autor.

Tabla 1. Herramientas facilitadoras de la Gestión del Conocimiento Socialización: Videoconferencia. Sistemas de conferencia virtual y Nonaka y Takeuchi asíncrona. Externalización: Correo electrónico. Listas de distribución,
(1995) (1995) Según
las interacciones que conforman el espiral Combinación: Correo electrónico. Herramientas de trabajo en grup. Tecnologias push o broadcast. Internalización: Herramientas de minería de datos basadas en redes neuronales. Simulaciones. Sistemas de Información geográfica. Bases de conocimientos. Formularios predefinidos para la carga de información. Integración entre las bases de datos. Internet. Estandarización

$\begin{array}{ll}\text { Johnson (1997) } & \text { de las aplicaciones ofimáticas. Capacidades de búsqueda sofisticadas. } \\ \text { Entornos de discusión colaborativos. Agentes inteligentes. }\end{array}$

$\begin{array}{ll}\text { Johnson (1997) } & \text { de las aplicaciones ofimáticas. Capacidades de búsqueda sofisticad } \\ & \text { Entornos de discusión colaborativos. Agentes inteligentes. }\end{array}$

Junnar
(1997)

Ruggles (1998)

Intranets - Extranets. Repositorios de conocimlento. Herramientas de ayuda a la toma de decisiones. Herramientas de trabajo en grupo.

Individuales: Para localizar información y conocimiento: Agrupación. Procesamiento del lenguaje natural. Extracción de resúmenes de textos. Bair y O'Connor Expansión semántica. Visualización. De Grupo: Para la reutilización del (1998) conocimiento dentro de un grupo y en la gestión de documentos en bases de datos. Corporativas: Para crear sistemas integrados de GC. (en tela de juicio).

Laudon y Laudon
(2000)
En función del uso
para cada área de
Gestión del
Conocimiento
Meso y Smith
(2000)
Según su función
en la GC

Compartición: Herramientas de groupware. Intranets. Distribución Crocesadore detera Herranientas de Procesadores de textos. Herramientas de autoedición y publicación en Web. Calendarios electrónicos. Bases de datos personales. Creación de conocimiento: Disefo asistido por ordenador (CAD). Realidad virtual Codificación y captura del conocimiento: Sistemas de experto. Inteligencia artificial. Redes neuronales, Lógica difusa. Algoritmos genéticos. Agentes Inteligentes.

Uso del conocimiento: Herramientas de trabajo en grupo. Videoconferencia

Tecnologias push. Tecnologías de apoyo a la toma de decisiones en grupo Búsqueda del conocimiento: Navegadores. Tecnologías Web. Herramientas de minerf́a de datos. Tecnologías de búsqueda y localización. Agentes inteligentes. Creación de conocimiento: Agentes inteligentes Empanuetado del conocimiento: Sistemas de gestión documental Agentes inteligentes.

Mentzas et at

- comunicación social: Correo electrónico. videoconferencias. Sistemas de mensajeria Como un plo Repositorios de conocimiento. Mapas de conocimiento. Intranets.

Fuente: Villagrasa (2011).

En resumen, se hace indiscutible la existencia de una correlación positiva entre las distintas fases de la gestión del conocimiento y la eficacia organizativa que conduce a la rentabilidad económica de las empresas, lo cual hace evidente que este escenario puede contribuir a paliar la situación financiera de las PYMES cuando éstas lo ameriten. De esta forma, la sapiencia de los gerentes, a través de prácticas comunes y complementarias, puede encontrar las diferentes modalidades de acceso al financiamiento de la PYMES, permitiendo esta visión, en todo caso, mejorar los niveles de competencia entre las organizaciones.

\section{Conclusiones}

Las PYMES en Venezuela se desenvuelven en medio de adversidades económicas producto de políticas de estado, con un gobierno que se afianza en las importaciones para abastecer el mercado interno, afectando en gran medida la producción nacional, al no crear planes estratégicos de fomento a la actividad económica interna. Estas organizaciones se de- baten en un punto de equilibrio para su supervivencia, requiriendo ser fortalecidas en el tiempo con técnicas y planes de financiamiento, tanto de organismos privados como públicos basados en la cooperación y alianzas estratégicas entre el estado - empresa privada - sectores financieros económicos, con el fin de sortear las incertidumbres y cambios que demanda el contexto actual. Estas estrategias deben además incluir planes de formación para la gerencia e incorporación de sistemas de redes e información, que les permita incorporarse efectivamente al mundo de la tecnología. Para que estos planes de fortalecimiento sean posibles y satisfacer las necesidades de financiamiento de las PYMES, es imprescindible poseer una gerencia con un alto estándar de conocimientos y cultura organizacional, para ser más eficaces y competitivas a la hora de requerir recursos financieros.

Las organizaciones en la búsqueda de estar a la par de los acontecimientos globales requieren estar permanentemente actualizadas y para ello deben disponer de una gerencia con un alto grado de cultura organizacional que le permita la generación de conocimiento y que entienda la realidad institucional en todos los ámbitos de la organización. Se hace indispensable, que el personal administrativo, operacional y financiero estén estrechamente unidos, alrededor de un pensamiento sistémico, y con interpretaciones del conocimiento desde la perspectiva de la conducta humana y el compromiso empresarial de todos los involucrados en la conducción de la organización, donde no exista limitación alguna para la búsqueda del conocimiento.

La gestión del conocimiento en las PYMES y las grandes industrias se basa en la trasferencia del conocimiento como un valor de la organización, tanto interna como externamente, bajo el esquema de intercambio de recursos y la fomentación de las relaciones y satisfacción de las de necesidades que coadyuven a la integración de toda la organización, con el fin de trabajar como una sola compañía con objetivos en común. La forma de gestionar el conocimiento dependerá de las características propias de cada PYME y al objetivo que quiera alcanzar.

Tanto la cultura organizacional como la gestión del conocimiento corporativo y disciplinado, conducen a que la gerencia tenga un mejor desempeño en la consecución de sus objetivos dentro de la administración financiera, pudiendo esta misma mejorar su capacidad de relacionarse, trabajar en equipo, afrontar la incertidumbre y si quieren ir más allá de los objetivos, deben abrir sus mentes en búsqueda de afrontar con éxito todos los elementos que constituyen a la gestión del conocimiento y con esta visión permitir mejorar los niveles de excelencia en la gestión de las PYMES, los cuales son necesarios en el acceso al financiamiento de estas empresas.

En definitiva, el reto es estructurar un plan nacional de apoyo y monitoreo constante de las PYMES en la adopción de políticas públicas integrales sostenibles, que abarquen la parte financiera alineada con la cultura organizacional y la gestión del conocimiento, con una gerencia proactiva, dinámica, enfocada en el manejo de las incertidumbres y la inestabilidad en un contexto global, dando identidad a su desempeño a través de los elementos de la cultura organizacional y gestionando el conocimiento como herramienta clave para, no sólo permanecer en el tiempo o sobrevivir, sino para ser competitivas y eficientes, pudiendo de esta forma acceder más fácilmente a oportunidades de financiamiento. Todo lo anterior, sugiere un compromiso invaluable de alcanzar el bienestar social y económico del país. 


\section{Referencias}

Aranguren, W. (2009). De la gerencia pública a la gerencia interorganizacional: un reto para la gestión gubernamental. Visión Gerencial, volumen (1), enero-junio 2009, pp. 5-23.

Barrigaz, N. y Bravo, M. (2012). Gestión del conocimiento como fuente transformadora en las organizaciones del siglo XXI. Observatorio de la Economía Latinoamericana, № 168, junio 2012.

Briceño, H. (2011). Reflexiones críticas sobre capital humano y diversidad cultural en las universidades venezolanas. Revista Estudios Gerenciales y de las Organizaciones, volumen (4), № 1, enero - junio 2011, pp. 177-190.

Buckingham, M. y Coffman, C. (1999). Primero, rompa todas las reglas. Colombia: Editorial Norma.

Canelones, O. (2014). Sociedades de garantías recíprocas regionales en Venezuela. Revista Orbis. Ciencias Gerenciales, año 10, No 29, noviembre 2014, pp. 174-193.

Cárdenas, A., González, E., González, A. y Marin, K. (2008). Herramientas financieras del mercado venezolano como alternativas de inversión. Revista Venezolana de Gerencia. Ciencias Gerenciales, año 13, № 44, pp. 573-594.

Celis, M. (2015). La conducta de las organizaciones. Bases para su estudio con una perspectiva sistémico-social. Valencia, Venezuela: Dirección de Medios y Publicaciones Universidad de Carabobo.

torial Norma.

Drucker, P. (2002). La Gerencia en la Sociedad Futura. Colombia: Edi-

Freitas, M. (1991). Cultura organizacional grandes temas en debate. Revista de Administração de Empresas, volumen (31), N № 3, pp. 73-82.

Galindo, L. (1999). Perspectiva actual de las fuentes de financiación en la empresa. España: Universidad de Cádiz.

Gálvez, E. y García, D. (2011). Cultura organizacional y rendimiento de las Mi PYMES de mediana y alta tecnología: un estudio empírico en Cali, Colombia. Cuadernos de Administración, volumen (24), N42, pp. 125-145.

García, C. (2006). Una Aproximación al Concepto de Cultura Organizacional. Universitas Psychologica, volumen (5), Nº1, enero-abril 2006, pp. 163 174.

Hernández, R. (2005). Epistemología y formación gerencial: un enfoque holístico. Revista Negotium. Ciencias Gerenciales, año 1, N 1, julio 2005, pp. 3-11.

Instituto Nacional de Estadísticas. (INE). Venezuela. Disponible en www. ine.gov.ve. Consulta en línea realizada el 17 de enero de 2018.

León, F. (2014). Teoría del conocimiento. Valencia, Venezuela: Dirección de Medios y Publicaciones Universidad de Carabobo.

Márquez, L. (1998). Gerencia de recursos humanos en tiempos de cambio. Debates IESA, año 4, N²

Mercado, H. y Palmerín, M. (2007). La internacionalización de las pequeñas y medianas empresas. México. Edición electrónica. Disponible en www. eumed.net/libros/2007c/334/

Morín, E. (2000). Los siete saberes necesarios para la Educación del futuro. Universidad Central de Venezuela / IESAL / UNESCO. Unidad de publicaciones. Caracas.

Muijca, M. (2010). Los Cambios Percibidos En El Ámbito De Las Ciencias Administrativas. Una Perspectiva Crítica. En Miguel Mujica (Coord), Estudio Contextualizado de la Administración en Venezuela (pp. 17-84). Valencia, Venezuela: Dirección de Medios y Publicaciones Universidad de Carabobo.

Nonaka, I. y Takeuchi, H. (1999). La Organización Creadora de Conocimientos. Cómo las compañías japonesas crean la dinámica de la innovación. EEUU: Oxford University Press.

Pedraja, L., Rodríguez, E. y Rodríguez, J. (2009). Gestión del conocimiento, eficacia organizacional en pequeñas y medianas empresas. Revista Venezolana de Gerencia, volumen (14), N 48, pp. 495-506.

Ramos, K. (2014). Valoración de las nuevas formas del comportamiento gerencial en Venezuela. Observatorio de la Economía Latinoamericana, № 200, julio 2014.

Romero, J. (2009). La formación de los gerentes en la perspectiva CTS. Revista Iberoamericana de Ciencia, Tecnología y Sociedad - CTS, octubre 2009. Sotomayor, R. (2004). Gerencia de Recursos Humanos en Venezuela. Revista Visión Gerencial, volumen (3), Año 3, No 2, julio - diciembre, 2004, pp. 80-83.

Valera, M. (2011). Dimensión humana bajo la óptica del cuadro integral en la gestión de los consejos comunales del estado Carabobo. Revista Estudios Gerenciales y de las Organizaciones, volumen (4), № 1, enero-junio 2011, pp. $65-83$.

Villagrasa, A. (2011). Gestión del conocimiento, pequeña - mediana empresa y nuevas prácticas gerenciales: un acercamiento teórico, en el contexto emergente. Revista Estudios Gerenciales y de las Organizaciones, volumen (4), $N^{\circ} 1$, enero-junio 2011, pp. 87-105. 